Relations industrielles

Industrial Relations

\title{
Les communistes et les unions ouvrières
}

\section{Gérard Dion}

Volume 2, numéro 9, mai 1947

URI : https://id.erudit.org/iderudit/1023877ar

DOI : https://doi.org/10.7202/1023877ar

Aller au sommaire du numéro

Éditeur(s)

Département des relations industrielles de l'Université Laval

ISSN

0034-379X (imprimé)

1703-8138 (numérique)

Découvrir la revue

Citer cet article

Dion, G. (1947). Les communistes et les unions ouvrières. Relations industrielles / Industrial Relations, 2(9), 1-2. https://doi.org/10.7202/1023877ar

Tous droits réservés @ Département des relations industrielles de l’Université Laval, 1947
Ce document est protégé par la loi sur le droit d'auteur. L’utilisation des services d'Érudit (y compris la reproduction) est assujettie à sa politique d'utilisation que vous pouvez consulter en ligne.

https://apropos.erudit.org/fr/usagers/politique-dutilisation/ 


\title{
Bulletin des Relations Industrielles
}

\author{
PUBLIE PAR LE DÉPARTEMENT DES RELATIONS INDUSTRIELLES \\ DE LA FACULTE dES SCIENCES SOCIALES DE LAVAL, QuÉbeC
}

T. R. P. G.-H. LEVESQUE, o.p., doyen - GERARD TREMBLAY, directeur - J. O'CONNELL-MAHER, assistant-direetour

Abbé GERARD DION, secrétaire - CHARLES BELANGER, assistant-secrétaíre

Vol. $2-N^{\circ} 9$

31 mai 1947

\section{LES COMMUNISTES ET LES UNIONS OUVRIÈRES}

La publicité qui a entouré de récents événements nous oblige à revenir sur un sujet que nous avons déjà traité l'an dernier, celui des communistes au sein des unions ouvrières canadiennes. Que des communistes se soient infiltrés au sein de certains syndicats, qu'ils en soient même devenus les dirigeants, c'est un fait reconnu et admis de tous. Il s'en trouve d'ailleurs dans beaucoup d'autres milieux. Cependant, notre position qui nous met en relations avec les groupements ouvriers tout autant qu'avec les groupements patronaux nous permet d'affirmer que ce ne sont encore là que des cas exceptionnels. Ce n'est pas parce que certains chefs ouvriers, dans des circonstances particulières, que nous nous abstenons de juger, emploient des méthodes assez radicales pour revendiquer ce qu'ils considèrent comme le droit des travailleurs que l'on est autorisé à crier au communisme. Soyons réalistes. Cela n'émeut pas du tout les syndiqués et bien plus, c'est faire une belle propagande aux communistes que de leur attribuer les avantages que les unions ouvrières réussissent à gagner pour leurs membres.

Il n'y a pas de doute que tout effort doit être tenté pour extirper des unions ouvrières canadiennes le germe du communisme et que la collaboration de toutes les bonnes volontés doit être obtenue à cette fin. Toutefois, nous nous permettons de signaler que, dans ce travail de collaboration, chacun doit prendre les moyens yui lui conviennent. Sinon, on obtiendra un effet contraire et au lieu de guérir le mal, on l'aggravera.

Mais avant tout, il est nécessaire de se demander comment il se fait qu'il y a du communisme et des communistes et comment ceux-ci ont réussi à prendre la direction de certaines unions ouvrières. Si l'on se donnait la peine de relire — ou même de lire - certains passages des documents pontificaux publiés depuis une quinzaine d'années, on se rendrait probablement compte de la clairvoyance des souverains pontifes et on trouverait peut-être aussi les vraies réponses à ces questions. En 1937, dans Divini Redemptoris, Pie XI avait dit: «Pour comprendre comment le communisme a réussi à se faire accepter sans examen par les masses ouvrières, il faut se rappeler que les travailleurs étaient déjà préparés à cette propagande par l'abandon religieux et moral où ils furent laissés par l'économie libérale... On recueille donc l'héritage des erreurs tant de fois dénoncées par nos Prédécesseurs et par Nous même. » Et plus loin, il ajoutait: «Il n'y aurait ni socialisme, ni communisme si les chefs des peuples n'avaient pas dédaigné les enseignements de l'Eglise et ses maternels avertissements. Mais ils ont voulu élever sur les bases du libéralisme et du laïcisme d'autres constructions sociales qui tout d'abord paraissaient puissantes et grandioses; mais on vit bientôt qu'elles n'avaient pas de fondements solides. » En 1931, dans Quadragesimo Anno, le même pontife avait déjà affrmé: «Nous ne jugeons assurément pas nécessaire d'avertir les fils bons et fidèles de l'Eglise touchant la nature impie et injuste du communisme, mais cependant Nous ne pouvons voir sans une profonde douleur l'incurie de ceux qui, apparemment insouciants de ce danger imminent et lâchement passifs, laissent se propager de toutes parts des doctrines qui, par la violence et le meurtre, vont à la destruction de la société tout entière. Ceuxlà surtout méritent d'être condamnés pour leur inertie qui négligent de supprimer ou de changer des états de choses qui exaspèrent les esprits des masses et préparent ainsi la voie au bouleversement et à la ruine de la société. » Le pape ne s'est pas contenté de diagnostiquer la cause du mal, il avait suggéré des remèdes: «Les moyens de sauver le monde actuel de la ruine dans laquelle le libéralisme amoral nous a plongés, ne consistent ni dans la lutte des classes ni dans la terreur, beaucoup moins encore dans l'abus autocratique du pouvoir de l'Etat, mais dans l'instauration d'un ordre économique inspiré par la justice sociale et les sentiments de la charité chrétienne. Nous avons montré comment une saine prospérité doit se baser sur les vrais principes d'un corporatisme sain... - et l'on sait que pour avoir du corporatisme, il faut avoir du syndicalisme. - La mission principale et la plus authentique du pouvoir civil est précisément de promouvoir efficacement cette harmonie et la coordination de toutes les forces sociales... Aussi, Nous Nous adressons tout particulièrement à vous, patrons et industriels, dont la tâche est souvent si difficile parce que vous portez le lourd héritage des fautes d'un régime économique injuste, qui a exercé ses ravages durant plusieurs générations; songez à vos responsabilités. Il est malheureusement trop vrai que les pratiques admises en certains milieux catholiques ont contribué à ébranler la confiance des travailleurs dans la religion de Jésus-Ouvrier. On ne voulait pas comprendre que la charité chrétienne exige la reconnaissance de certains droits qui appartiennent à l'ouvrier et que l'Eglise lui a explicitement reconnus... Que dire de ces industriels catholiques qui n’ont cessé jusqu'à présent de se montrer hostiles à un mouvement ouvrier que Nous avons Nous même recommandé ? N'est-il pas déplorable qu'on ait parfois abusé du droit de propriété, reconnu par l'Eglise, pour fruster l'ouvrier du juste salaire et de ses droits sociaux qui lui reviennent ?»

Ces paroles de Pie XI sont claires et précises. On ferait bien de les méditer sérieusement. Il y trace un (Suite à la dernière page) 


\section{LA FÉDÉRATION DES COMITÉS PARITAIRES}

La Fédération des comités paritaires de la province de Québec est née, à l'automne de 1937, de la pensée généreuse de secrétaires de comités paritaires de la région de Montréal soucieux d'assumer toutes leurs responsabilités sociales et de travailler en communion d'idées, de concert avec le ministère du Travail, pour tout ce qui se rapporte à l'application des clécrets et à la gestion des comités paritaires. L'inspiration qui a présidé à sa naissance est donc des plus louable et ne saurait être que féconde. Nullement limité à une région déterminée, le champ de son extension territoriale est à l'échelle de la province. La Fédération ne fait exception d'aucun comité paritaire. Au contraire, elle les invite tous à participer à ses activités; en fait, elle voudrait les voir tous joindre ses rangs.

Conformément à sa constitution, la Fédération a particulièrement pour mission:

-De promouvoir les intérêts de tous les comités paritaires de la province et de resserrer davantage les liens professionnels qui unissent leurs secrétaires afin de les amener à échanger les fruits de leurs expériences et à collaborer plus étroitement entre eux sur tous les problèmes d'intérêt commun et particulier;

-D'agir en qualité de conseiller tant auprès du ministère du Travail qu'auprès de chaque comité paritaire pour toutes questions pertinentes à l'application des décrets passés en exécution de la Loi de la convention collective:

-D'assister les parties contractantes qui désirent l'extension aux tiers de la convention collective de travail qu'elles ont conclue;

-De communiquer aux secrétaires des comités paritaires toute information qui pourrait leur être de quelque utilité et, par là, de faire oeuvre d'entr'aide;

-Enfin, de promouvoir l'extension juridique des conventions collectives de travail qui, dans l'économie actuelle des relations industrielles, représentent la meilleure formule pour l'aménagement de stables, saines et fécondes relations entre le capital et le travail.

On en conviendra, c'est là un magnifique programme. Mais la Fédération n'a pas fait que se fixer des objectifs, elle est passée aux réalisations.

Sans vouloir décrire toute l'activité qu'elle a déployée depuis son existence, limitons-nous à énumérer les initiatives prises en ces derniers mois ou présentement en cours.

La Fédération a fait tenir au ministère du Travail, sur demande expresse de ce dernier, un Mémoire de vingt-six amendements à la Loi de la convention collective aux fins de rendre ctte loi plus souple et plus logique dans son application. C'est d'ailleurs l'intention du ministère du Travail que dorénavant les amendements désirés à la susdite loi lui viennent pour une bonne part par l'intermédiaire de la Fédération.

La Fédération a revisé sa constitution et ses règlements afin de les mettre au point. Pour éclairer l'opinion publique sur les dispositions de la Loi de la convention collective et signaler les avantages économiques et sociaux qui en découlent, elle collabore au Bulletin des Relations industrielles par une chronique mensuelle. Elle étudie l'opportunité et la possibilité d'uniformiser les livrets d'apprentissage et la carte d'identité pour tous les inspecteurs des comités paritaires. Elle s'est chargée de l'étude du problème d'un fonds de pension pour les employés des comités paritaires. Enfin, elle a entrepris, en vue de réaliser une oeuvre de plus large envergure, d'organisr un centre de documentation et d'information à l'usage des secrétaires des comités paritaires.

Voilà un bilan d'activités qui, à lui seul, suffirait à justifier on ne peut mieux l'existence de cette Fédération.

La Fédération des comités paritaires de la province de Québec sait par les nombreuses marques de bienveillance qu'il lui a témoignées que le ministère du Travail met beaucoup d'espérance dans la réalisation des fins qu'elle s'est données et qu'elle poursuit, et veut poursuivre avec plus d'intensité si possible. Elle ne redoute nullement, grâce à l'appui extérieur qui lui est prêté, que des influences étrangères en viennent à s'ingérer dans l'organisation de sa vie interne.. La Fédération est un corps professionnel organisé dans l'Etat selon le principe de la fonction supplétive des communautés et le rôle de l'Etat n'est pas de détruire ou d'absorber les groupements et les institutions qui sont les nécessaires intermédiaires entre l'individu et la société civile, mais de les aider à s'épanouir et de favoriser leur essor dans le respect de leur autonomie

La présence de l'honorable ministre du Travail, ainsi que de celle des sous-ministres du Travail, à son assemblée générale mensuelle d'avril, tenue à Québec, a marqué un tournant dans l'histoire de la Fédération. Cette organisation est officiellement campée devant l'opinion publique. Après quelque dix ans d'existence, voici qu'elle a dépassé le stage de l'organisation. Elle entre maintenant dans une ère nouvelle avec droit de cité au milieu des autres associations professionnelles.

Marcel FORGET

\section{LES COMMUNISTES ET LES UNIONS OUVRIERES}

(Suite de la première page)

programme d'action pour tous ceux qui veulent le triomphe de la civilisation chrétienne en notre pays. Les employeurs ont sans doute un rôle à remplir dans ce domaine, même il y a des choses qui ne peuvent être accomplies que par eux. Avant de faire quoi que ce soit, qu'ils commencent par examiner leur conscience, qu'ils songent qu'il $\epsilon$ st en leur pouvoir d'attaquer le communisme à sa base en opérant des réformes dans leurs relations avec leurs employés. Qu'ils considèrent partout et toujours leurs ouvriers comme des hommes, qu'ils leur accordent des salaires raisonnables et des conditions de travail humaines. Ensuite, qu'ils ne s'acharnent pas à lutter contre les syndicats ouvriers, sous quelque prétexte que ce soit. C'est de cette façon quilis rendront les plus grands services à la cause. Mais, en définitive, la lutte contre les communistes au sein des unions ouvrières ne pourra se faire efficacement que par les intéressés eux-mêmes. On sait que toute coercition extérieure ne peut que produire des effets passagers et superficiels. Il va donc falloir que tous les travailleurs, et surtout ceux qui ont la prétention de se considérer comme les meilleurs, mettent de côté leur indi- vidualisme, qu'ils s'occupent de leurs affaires, qu'ils adhèrent aux syndicats ouvriers. Aussi longtemps qu'une bonne partie d'entre eux laisseront «aux autres 》 le soin d'aménager les relations du travail, qu'ils seront trop négligents pour se donner la peine d'assister aux réunions de syndicats ou s'instruire des problèmes ouvriers, qu'ils se récuseront à accepter des postes qui demandent du travail et des sacrifices, les communistes auront beau jeu pour accéder facilement à des charges importantes dans les unions ouvrières, sous quelque nom qu'elles se présentent et à quelque fédération qu'elles appartiennent. Une minorité de gens actifs qui agissent dans un but précis aura toujours le dessus sur une masse insouciante et ignorante.

A chacun donc sa tâche et ses responsabilités. Moins d'agitation, plus de justice et de charité, ce sera probablement plus efficace. Une revue américaine écrivait dernièrement sur ce problème: "No anti-Communist employer is as anti-Communist as the labor leaders who have fought Communists in their own unions. Employers - and politicians - would be well advised to keep out of this fight and leave it to people who, when they are talking about Communists, know what they are talking about».

Gérard Dion 